\title{
De Novo Granulocytic Sarcoma of the Humerus in an 82-Year-Old Woman: A Case Report
}

\author{
Hiroyuki Nagasawa ${ }^{\text {a, }}$, Kyoji Okada ${ }^{\mathrm{b}}$, Naohisa Miyakoshi ${ }^{\mathrm{a}}$, Hiroyuki Tsuchie ${ }^{\mathrm{a}}$, \\ Hiroshi Nanjo ${ }^{\mathrm{c}}$, Yoichi Shimada ${ }^{\mathrm{a}}$
}

\begin{abstract}
Granulocytic sarcoma is a rare solid malignant tumor that occurs in patients with acute myeloid leukemia. As such, granulocytic sarcoma without leukemia occurring in long bones is quite rare. This case report describes an isolated granulocytic sarcoma of the right humerus in an 82-year-old woman in the absence of acute myeloid leukemia. The patient was admitted to our hospital with a pathological fracture of the right humerus. An incisional biopsy was performed, and the pathological diagnosis was granulocytic sarcoma. Radiotherapy (45 Gy) to the humerus resulted in a good clinical outcome with no evidence of myeloid leukemia at 9.5 years of follow-up. Radiotherapy was effective for pain relief and maintenance of good quality of life in this patient. Although laboratory evaluation has not revealed any abnormalities 9.5 years after radiotherapy, periodic observation is required.
\end{abstract}

Keywords: Granulocytic sarcoma; Humerus; Diagnosis; Radiotherapy

\section{Introduction}

Granulocytic sarcoma (GS), also called extramedullary acute myeloid leukemia (AML) or myeloid sarcoma or chloroma, has been defined as a tumor of immature granulocytes, monocytes, or both occurring in extramedullary sites. GS is a rare malignant tumor that occurs in association with AML in $2-8 \%$ of cases $[1,2]$. GS that occurs in patients without leukemia has been called "de novo" or "isolated" GS. GS often arises in

Manuscript submitted June 12, 2020, accepted June 23, 2020

Published online July 21, 2020

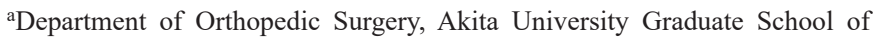
Medicine, Akita, Japan

bDepartment of Physical Therapy, Akita University Graduate School of Health Science, Akita, Japan

'Department of Clinical Pathology, Akita University Hospital, Akita, Japan

${ }^{\mathrm{d} C}$ Corresponding Author: Hiroyuki Nagasawa, Department of Orthopedic Surgery, Akita University Graduate School of Medicine, 1-1-1 Hondo, Akita 0108543, Japan. Email: nagasawa@med.akita-u.ac.jp

doi: https://doi.org/10.14740/jmc3526 several organs, including the skin, soft tissue, bones and lymph nodes. In contrast, de novo GS occurring in long bones is extremely rare. In this report, we describe the clinical, radiological and histological findings of an 82-year-old female with de novo GS in the humerus.

\section{Case Report}

An 82-year-old woman with a history of chronic thyroiditis experienced acute right upper arm pain when she grasped a bus handrail. She was admitted to another hospital, and a pathological fracture of the shaft of the right humerus was observed in radiographs. Although the patient underwent a systemic survey, no malignant lesions were identified. She presented to our hospital 2 months after the onset of pain. Radiographs showed a relatively demarcated osteolytic lesion with a pathological fracture of the right humerus. On magnetic resonance imaging (MRI), T1-weighted images showed a both intra- and extramedullary lesion with low signal intensity (Fig. 1a). On T2-weighed images, the mass showed high signal intensity (Fig. 1b). The lesion was irregularly and moderately enhanced after administration of gadolinium (Fig. 1c). No abnormalities were observed on routine blood testing, except for a slightly elevated serum soluble interleukin-2 receptor level $(824 \mathrm{U} / \mathrm{mL})$. The white blood cell count was $8,000 / \mathrm{mm}^{3}$, and the leukocyte differential was: $61.3 \%$ neutrophils, $2.5 \%$ eosinophils, $0.3 \%$ basophils, $6.0 \%$ monocytes, $29.9 \%$ lymphocytes and $0 \%$ blast cells. Positron emission tomography with ${ }^{18} \mathrm{~F}$-fluorodeoxyglucose (FDG-PET) showed a high maximum standardized uptake value $\left(\mathrm{SUV}_{\max }\right)$ in the right humerus and thyroid gland (Fig. 2). Fine-needle aspiration cytology of the thyroid gland revealed chronic thyroiditis without atypical cells.

Incisional biopsy of humerus was performed. Pathological examination of the biopsied specimen revealed an extensive growth of small, round, premature tumor cells in the presence of a few mitotic figures. Immunohistochemical analysis revealed strong positivity for CD68 (Fig. 3), but negativity for myeloperoxidase (MPO). Cytogenetic analysis of bone marrow cells revealed a normal karyotype with hypocellular marrow. The final diagnosis was monoblastic GS.

The patient underwent radiotherapy (RT) at a dose of 45 Gy. She did not receive any anti-leukemic chemotherapy after RT, since her arm pain was reduced and laboratory evaluation was not revealing. The patient remains alive at 9.5-year 


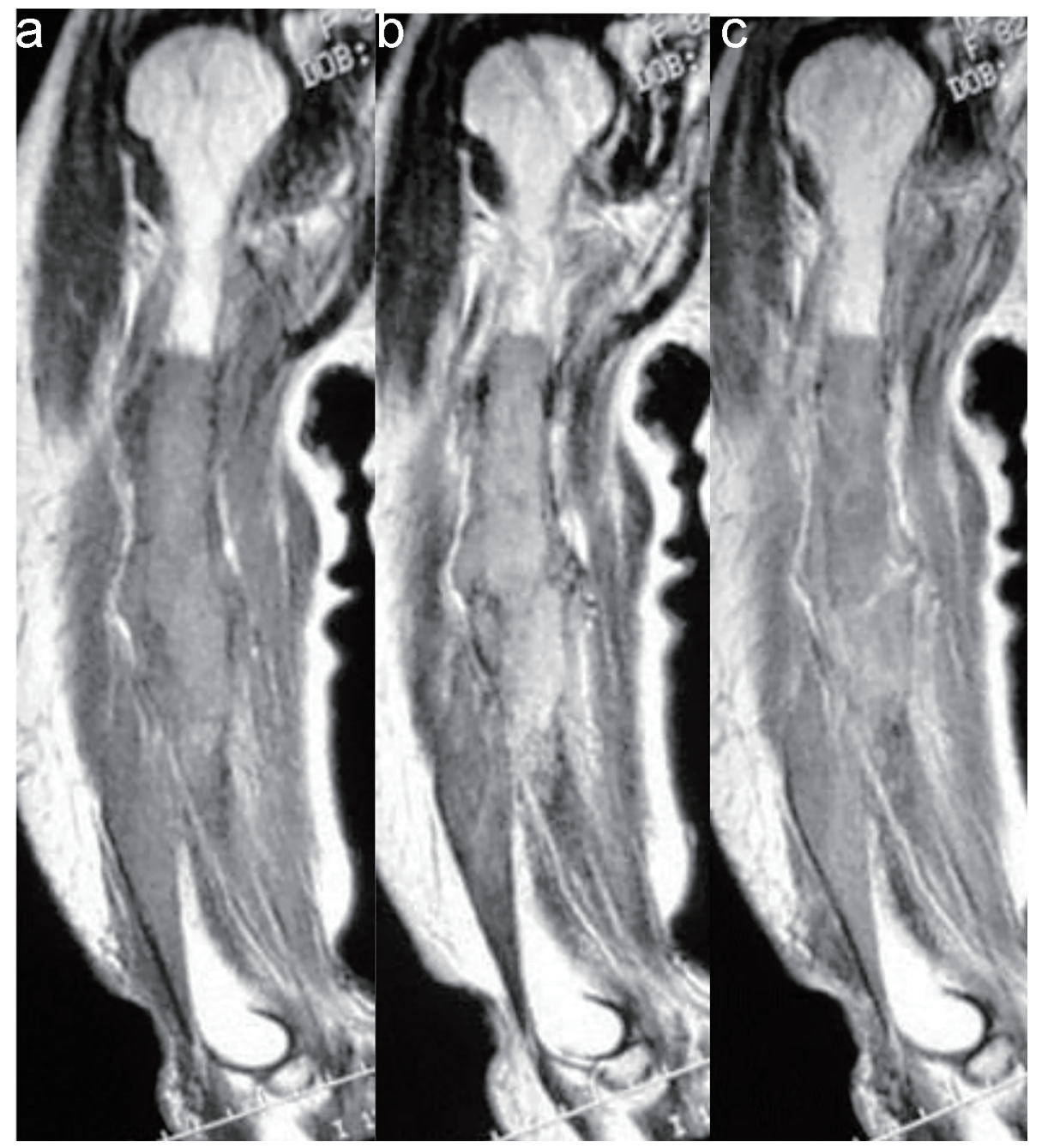

Figure 1. (a) Coronal T1-weighted MR image of the right humerus demonstrated both intra- and extramedullary lesion with low signal intensity. (b) Coronal T2-weighted MR image revealed a lesion with high signal intensity. (c) The lesion showed irregular and moderate enhancement after administration of gadolinium. MR: magnetic resonance.

follow-up, without any signs of AML or local recurrence in the humerus.

\section{Discussion}

According to the 2008 World Health Organization (WHO) classification, GS is defined as a tumor mass consisting of myeloid blasts or immature myeloid cells, occurring in an extramedullary site [3]. GS is divided into three types: 1) GS associated with AML in patients who already have AML or preceding AML; 2) GS associated with chronic myeloproliferative disorder (CMPD) and myelodysplastic syndrome (MDS); and 3) de novo GS [3]. GS occurs at any age but is most common in young adults and children. In a review of 74 patients with GS, the median age at diagnosis was reported to be 32 years in males and 34 years in females [4]. Thus, the present case was much older than typical cases. Untreated GS progresses to AML within months (median, 3 - 9 months), and the median survival period after progression to AML has been reported to be 6 - 14 months [5].

Woodard et al reported that bone is affected in $10-12.5 \%$ of GS cases, and the most commonly affected sites are in the axial skeleton, including the skull, vertebrae, sternum and ribs [6]. However, including the current case, only four reports of GS in long bones have been published in the English literature [6-8]. Khunger et al reported the case of GS in the humerus that occurred in a 20-year-old male; however, blast cells were observed in the peripheral blood of this patient [7]. Woodard et al also reported a case of GS in the humerus in a 31-year-old male with eosinophilia (white blood count $21,500 / \mathrm{mm}^{3}$, and a leukocyte differential revealing $63 \%$ mature eosinophils) [6]. Furthermore, "de novo" GS in long bones is extremely rare [8]. Rodriguez Perez et al described a case of isolated granulocytic sarcoma occurring in the humerus in a 69 -year-old woman in the absence of leukemia [8]. It should be stressed that in all four of these cases, the humerus was affected, and that all of the "de novo" cases occurred in relatively elderly patients. Ap- 


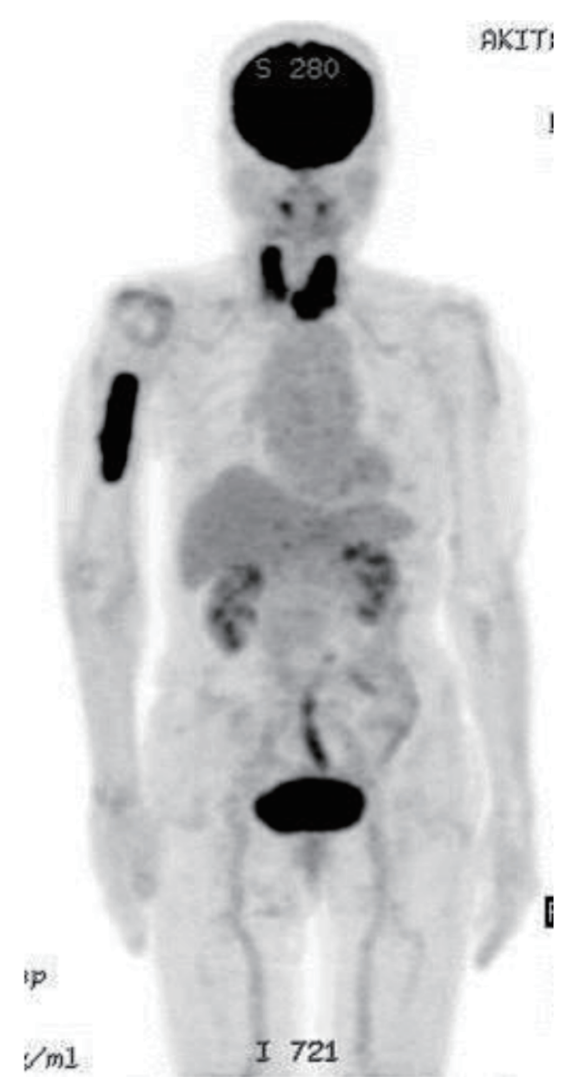

Figure 2. Positron emission tomography with ${ }^{18} \mathrm{~F}$-fluorodeoxyglucose (FDG-PET) demonstrated the high maximum standardized uptake value $\left(S_{U V} V_{\max }\right)$ in the right humerus and bilateral thyroid glands.

proximately $50 \%$ of GS cases are initially misdiagnosed, most frequently as lymphoma $[4,9]$, as well as small round cell tumors, undifferentiated carcinoma or melanoma, extramedullary localization CMPD, or other non-malignant lesions [10]. Immunohistochemical analysis is useful for preventing misdiagnosis. Audouin et al proposed that some variants of GS could be distinguished based on by immunohistochemical analysis of MPO, CD68/KP1/PGM1, lysozyme, CD34, factor VIII, CD61 and $\mathrm{CD} 31$ as follows: granulocytic (MPO+, CD68+ (KP1 PGM1-), lysozyme+, CD34 \pm ), monoblastic (MPO-, CD68+ (KP1+, PGM1+), lysozyme+, CD34-), myelomonoblastic (MPO-, CD68+ (KP1+, PGM1+), lysozyme+, CD34-), or megakaryoblastic (positivity for factor VIII, CD61, CD31) [10].

Several reports about GS treatment, including surgical resection, chemotherapy and RT, and their clinical outcomes have been reported. Yamauchi et al reported that the non-leukemic period after diagnosis of GS was significantly longer in patients who received chemotherapy compared to patients only underwent biopsy, surgical resection, or local RT of the tumor [4]. Tsimberidou et al reported that among 1,720 AML patients and 23 GS patients, anti-AML therapy was highly effective in patients with non-leukemic GS, and suggested the necessity of AML-type chemotherapy for patients with non-leukemic GS [11]. However, information about optimal treatment for de novo GS has been limited, and it is still not clear whether the addition of RT results in a superior overall outcome compared

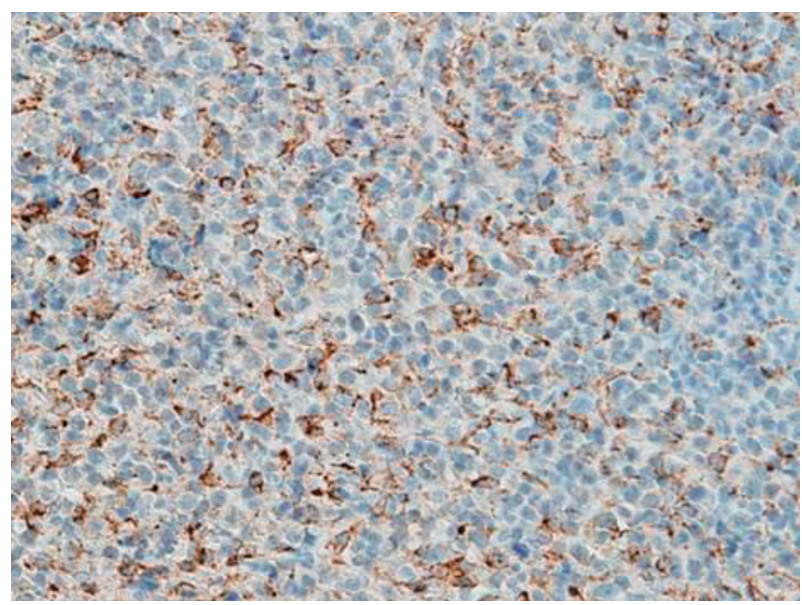

Figure 3. Photomicrograph of the immunostained humerus tumor showed CD68-positive tumor cells.

with chemotherapy alone in patients with GS involving bone [12]. Bakst et al analyzed 22 cases of GS who underwent RT and suggested that a low-dose regimen of 24 Gy in 12 fractions using conventional treatment can be used in the majority of cases with excellent disease control and minimal morbidity [13]. Chen et al reviewed 20 GS patients with 43 lesions, and observed that the complete remission rate is optimal using moderate RT doses between 20 and 30 Gy with conventional fractionation [14]. In the current case, the patient received only RT (45 Gy) based on her age, and local control has been achieved without any complaints. However, for GS occurring in long bones, there is no report on the possibility of developing AML and the time of onset in patients treated with only RT.

\section{Conclusion}

In conclusion, we have presented an 82-year-old female with a rare variant of isolated GS occurring in the humerus. Histological and immunohistochemical examinations are required for diagnosis in such de novo GS cases. RT was effective for pain relief and maintenance of good quality of life in this patient. Although laboratory evaluation has not revealed any abnormalities 9.5 years after RT, periodic observation is required.

\section{Acknowledgments}

None to declare.

\section{Financial Disclosure}

None to declare.

\section{Conflict of Interest}

None to declare. 


\section{Informed Consent}

Not applicable.

\section{Author Contributions}

Hiroyuki Nagasawa collected the clinical data and drafted the manuscript. Naohisa Miyakoshi, Hiroyuki Tsuchie and Yoichi Shimada reviewed this manuscript. Kyoji Okada and Hiroshi Nanjo reviewed the pathological findings of this case.

\section{Data Availability}

The authors declare that data supporting the findings of this study are available within the article.

\section{References}

1. Muss HB, Moloney WC. Chloroma and other myeloblastic tumors. Blood. 1973;42(5):721-728.

2. Liu PI, Ishimaru T, McGregor DH, Okada H, Steer A. Autopsy study of granulocytic sarcoma (chloroma) in patients with myelogenous leukemia, Hiroshima-Nagasaki 1949-1969. Cancer. 1973;31(4):948-955.

3. Pileri SA, Orazi A, Falini B. Myeloid sarcoma. World Health Organization classification of tumours of haematopoietic and lymphoid tissues. Lyon, France: IRAC press. 2008; p. 140-142.

4. Yamauchi K, Yasuda M. Comparison in treatments of nonleukemic granulocytic sarcoma: report of two cases and a review of 72 cases in the literature. Cancer. 2002;94(6):1739-1746.

5. Imrie KR, Kovacs MJ, Selby D, Lipton J, Patterson BJ,
Pantalony D, Poldre P, et al. Isolated chloroma: the effect of early antileukemic therapy. Ann Intern Med. 1995;123(5):351-353.

6. Woodard WL, 3rd, Kneisl JS, Lipford EH. Isolated granulocytic sarcoma of the humerus. Orthopedics. 1999;22(8):773-775.

7. Khunger JM, Sharma MP, Talib VH. Granulocytic sarcoma of humerus, an unusual association of acute myeloblastic leukemia - a case report. Indian J Pathol Microbiol. 2000;43(4):475-478.

8. Rodriguez Perez A, Lopez Carrizosa MC, Villalon Blanco L, Samper Ots PM, Ortiz Cruz E. Granulocytic sarcoma of the right humerus in a non-leukaemia patient. Clin Transl Oncol. 2008;10(11):758-760.

9. Byrd JC, Edenfield WJ, Shields DJ, Dawson NA. Extramedullary myeloid cell tumors in acute nonlymphocytic leukemia: a clinical review. J Clin Oncol. 1995;13(7):1800-1816.

10. Audouin J, Comperat E, Le Tourneau A, Camilleri-Broet S, Adida C, Molina T, Diebold J. Myeloid sarcoma: clinical and morphologic criteria useful for diagnosis. Int J Surg Pathol. 2003;11(4):271-282.

11. Tsimberidou AM, Kantarjian HM, Estey E, Cortes JE, Verstovsek S, Faderl S, Thomas DA, et al. Outcome in patients with nonleukemic granulocytic sarcoma treated with chemotherapy with or without radiotherapy. Leukemia. 2003;17(6):1100-1103.

12. Bakst RL, Tallman MS, Douer D, Yahalom J. How I treat extramedullary acute myeloid leukemia. Blood. 2011;118(14):3785-3793.

13. Bakst R, Wolden S, Yahalom J. Radiation therapy for chloroma (granulocytic sarcoma). Int J Radiat Oncol Biol Phys. 2012;82(5):1816-1822.

14. Chen WY, Wang $\mathrm{CW}$, Chang $\mathrm{CH}$, Liu $\mathrm{HH}$, Lan $\mathrm{KH}$, Tang JL, Tien HF, et al. Clinicopathologic features and responses to radiotherapy of myeloid sarcoma. Radiat Oncol. 2013;8:245. 